\title{
KU activity: a method for calculating histopathologists' workloads
}

\author{
S K Suvarna, M S Kay
}

\begin{abstract}
An attempt to model activity of individual histopathologists or departments is illustrated, in which specimen numbers and types are weighted to produce an overall Kim unit (KU) activity. By cross referencing this numerical score against individual pathologists and clinicians, it is not only possible to interrogate departmental activity retrospectively, but also to predict and cost the impact of any change in service. The advantages over Welcans and Korner Units are illustrated.

(f Clin Pathol 1998;51:530-534)
\end{abstract}

Keywords: histopathology; workload; activity analysis

Various methods of calculating departmental workloads within histopathology have been tried, previously with limited satisfaction. Too often the information gathered tells one little about previous departmental activity and has nothing to say in terms of predicting the future. Given that Korner units ${ }^{1}$ and Welcans ${ }^{2}$ have limitations in analysing medical workload, an alternate methodology was sought. In this paper we describe the Northern General Hospital histopathology department's solution to this dilemma, introducing the KU (Kim unit, pronounced " $Q$ ") analysis, based on individual histopathologists' work, and we highlight the predictive power of this analysis.

\section{Methods}

Given that the range of specimens arriving in the average histopathology department is wide, our initial task was to limit the specimen coding when "booking in" to a standard number of relevant types and to put any further data into a non-coding part of the field in parentheses. Examples would include; gastric biopsy (cardia), lung (left lower lobe), or lymph node (digastric). The method of data capture also had to reflect specimens in which several parts were included with the main piece of tissue. An example might be a Whipple's resection that would include the pancreas with a cuff of duodenum, the spleen, and two separate lymph node groups. This would be coded as four specimens:

(1) Pancreas (with duodenum)

(2) Spleen

(3) Lymph node (coeliac axis group)

(4) Lymph node (inferior mesenteric group) This method allows the case load received to be split into defined groups of specimens and yet allows the subparts-generating work in addition to the main part of the specimen-to be identified and assessed appropriately.
Table 1 Examples indicating how the original unit of weighting was derived

\begin{tabular}{llrll}
\hline \multirow{4}{*}{ Sample } & \multicolumn{2}{l}{ Times } & & \\
\cline { 2 - 4 } & Dissection & Microscopy & Reporting & \multirow{2}{*}{ KU } \\
\hline Appendix & 2 & 2 & 1 & 1 \\
Gastric biopsy & 1 & 4 & 2 & 2 \\
Hemicolectomy & 7 & 5 & 3 & 3 \\
Mastectomy & 7 & 8 & 5 & 4 \\
Kidney biopsy & 1 & 20 & 8 & 5 \\
Aspirate (pleura) & 0 & 2 & 1 & 1 \\
\hline
\end{tabular}

The individual specimen types were then assigned (by the consultant staff body as a whole) a difficulty quotient/weighting on a scale of $1-5$ units (KU), depending on the extent of the following manipulations:

- Time for dissection

- Time for macroscopic description

- Number of sections/stains required

- Complexity of specific tests, for example electron microscopy

- Time for microscopic diagnosis reflecting the number of sections and stains

- Time to dictate a report

A few examples are given in table 1 indicating how the original unit of weighting was derived.

Alternate ranges of KU activity were considered. A smaller range of 1-3 KU was felt to be insufficient for the range and complexity of the cases that the department was receiving; $5 \mathrm{KU}$ was considered to be simple and yet allowed clear stratification of the specimen types in terms of their weighting. The possibilities of a wider range -7 and $10 \mathrm{KU}$ - were also considered. It is possible to do the same analysis with these ranges but no inherent advantages were seen, since any larger range just produces bigger scores. The time for specimen dissection, microscopy, and reporting these biopsies was based upon an "average" case. A complete listing of the weightings derived is given in table 2 .

To make the analysis easier to follow, groups of specimens were aligned together, thereby providing clearer insight into the numerical scores of the specimens sent to the department. For example "small surgical biopsies" include: - Oesophageal biopsy

- Gastric biopsy

- Duodenal biopsy

- Small intestinal biopsy

- Colonic biopsy

- Rectal biopsy

- Anal biopsy

Subdivision of these specimens reflects clinical directorates at this centre and any alternate structural layout of the information would be permissible for different centres.

The numerical totals of the specimens received can thus be displayed in groups
Accepted for publication 27 March 1998 
Table 2 Complete list of $K U$ weightings

\begin{tabular}{lllr}
\hline Anal biopsy & 1 & Oesophagus & 3 \\
Anus & 1 & Omentum & 1 \\
Appendix & 1 & Other & 2 \\
Bladder & 4 & Ovary & 2 \\
Bladder biopsy & 1 & Pancreas & 2 \\
Blood vessel & 1 & Placenta & 1 \\
Bone & 3 & Pleura & 3 \\
Bone marrow & 3 & Pleural biopsy & 3 \\
Breast biopsy & 3 & Peritoneum & 1 \\
Bronchial biopsy & 1 & Prostatic chippings & 2 \\
Cardiac valve & 1 & Rectal biopsy & 1 \\
Cervical biopsy & 1 & Research & 1 \\
Colonic biopsy & 1 & Salivary gland & 1 \\
Duodenal biopsy & 1 & Skin & 2 \\
Endometrial biopsy & 1 & Small bowel & 3 \\
Explanted heart & 4 & Soft tissue & 3 \\
Fallopian tube & 1 & Spleen & 2 \\
Fetus & 4 & Stomach & 4 \\
Gall bladder & 1 & Testis & 2 \\
Gastric biopsy & 2 & Thymus & 3 \\
Hemicolectomy & 3 & Thyroid & 2 \\
Jejunal biopsy & 1 & Transplant kidney biopsy & 5 \\
Joint tissue & 2 & Uterus and cervix & 2 \\
Kidney & 5 & Uterus, tubes and ovaries & 3 \\
Kidney biopsy & 5 & Vagina & 2 \\
Liver biopsy & 4 & Vaginal biopsy & 1 \\
LLETZ/cone biopsy & 3 & Vas deferens & 1 \\
Lung & 3 & Vulval biopsy & 1 \\
Lymph node & 2 & Vulvectomy & 3 \\
Mastectomy/breast & 4 & Wertheim's & 4 \\
Mouth & 1 & Cervical cytology & 2 \\
Myocardial biopsy & 3 & Non-gynaecological cytology & \\
Nerve & 1 & Necropsy & \\
Oesophageal biopsy & 1 & Supervision of junior's specimen report & 2 \\
\hline & & & 2 \\
\hline
\end{tabular}

according to the pathologist examining them. Beneath this the total surgical weighting is expressed in terms of $\mathrm{KU}$, and beneath this line the more straightforward calculations for nongynaecological cytology, cervical cytology, supervised junior cases, and necropsies may be seen, with $1,2,2$, and $10 \mathrm{KU}$ values assigned per specimen.

A 12 month analysis is presented in table 3 from figures at our site. In addition, we have also tabulated the total specimens, KUs, and Welcans for the individual pathologists in terms of histology, non-gynaecological cytology, cervical cytology, and necropsies in terms of their numerical scores and weighted scores, together with percentage breakdowns (table 4). Furthermore, a comparison with Korner units is also provided in terms of the total output of the laboratory in table 4 .

\section{Results}

For the 12 month period in question, this department dealt with 13055 surgical cases, 35433 cervical cytology cases, 1692 nongynaecological cytology cases, and 384 necropsies.

Applying the KU numerical methodology yielded the results in table 3 . We felt this analysis and specimen grouping yielded a clear picture of the department's workload across the differing areas of histopathology. It also permitted individual consultants' work patterns to be viewed in relation to their colleagues and the department as a whole. We were pleased to see the pattern emerging that was consistent with our personal evaluation of the work of the department and were similarly pleased that, having discussed the methodology in detail beforehand, no conflicts emerged with the production of the figures.

According to the totalled results (table 5) for specimens, KUs, Welcans, and Korner units, the department appears to behave in different ways. The relative weighting of histology samples appears broadly comparable for specimen numbers, KUs, and Korner units, but Welcans clearly underestimate the volume of this work in the department.

Welcans similarly overemphasise the relative impact of cervical cytology in our department. There is also seen to be a difference in the specimen numbers and the values described by Korner units and KUs for cervical cytology. Members of the department consider that the KU figure most closely approximates the impact of cervical cytology on pathologists.

Non-gynaecological cytology provides a similar wide range, depending on which value one uses for result expression. The KU figure was judged to be most accurate, with the specimen count and Korner value apparently producing over- or underestimates of this workload fraction.

The same arguments may be applied to the necropsy column. The specimen numbers are obviously low, although the majority of this workload comprises coroner's necropsies de-

Table 3 Specimen workload (one year) for seven histopathologists $(A-G)$

\begin{tabular}{|c|c|c|c|c|c|c|c|c|}
\hline & $A$ & $B$ & $C$ & $D$ & $E$ & $F$ & $G$ & Total \\
\hline Small biopsy & 248 & 65 & 537 & 773 & 864 & 446 & 841 & 3774 \\
\hline Surgical (medium) & 180 & 20 & 325 & 207 & 293 & 255 & 432 & 1712 \\
\hline Surgical (large) & 22 & 13 & 29 & 25 & 52 & 55 & 68 & 264 \\
\hline Endocrine & 33 & 10 & 21 & 10 & 30 & 44 & 23 & 171 \\
\hline Lymphoreticular & 34 & 7 & 121 & 62 & 211 & 86 & 70 & 591 \\
\hline Cardiac & 152 & 0 & 43 & 44 & 178 & 30 & 23 & 470 \\
\hline Orthopaedic & 12 & 0 & 21 & 16 & 67 & 17 & 48 & 181 \\
\hline Male & 0 & 0 & 0 & 1 & 0 & 4 & 131 & 136 \\
\hline Female biopsy & 143 & 572 & 561 & 309 & 436 & 273 & 441 & 2735 \\
\hline Female (mid) & 65 & 0 & 100 & 58 & 126 & 114 & 195 & 658 \\
\hline Female (large) & 37 & 0 & 109 & 41 & 56 & 48 & 89 & 380 \\
\hline Skin & 380 & 3 & 729 & 619 & 909 & 698 & 971 & 4309 \\
\hline Special biopsy & 12 & 0 & 15 & 239 & 81 & 10 & 33 & 390 \\
\hline Respiratory & 103 & 0 & 40 & 35 & 123 & 66 & 53 & 420 \\
\hline ETC/other & 240 & 0 & 31 & 40 & 104 & 0 & 4 & 428 \\
\hline Total specimens & 1670 & 690 & 2682 & 2479 & 3530 & 2146 & 3422 & 16619 \\
\hline Histology KUs & 3252 & 819 & 5553 & 5871 & 7983 & 4771 & 6497 & 3474 \\
\hline Supervision KUs & 280 & 230 & 864 & 1640 & 1192 & 990 & 0 & 5196 \\
\hline Cervical cytology KUs & 0 & 3682 & 2276 & 0 & 808 & 896 & 292 & 7954 \\
\hline Non-gynaecology KUs & 3 & 1207 & 32 & 1 & 375 & 123 & 115 & 1856 \\
\hline Necropsy KUs & 300 & 0 & 720 & 840 & 760 & 610 & 610 & 3840 \\
\hline Total KUs & 3835 & 5938 & 9445 & 8352 & 11118 & 7390 & 7514 & 53592 \\
\hline
\end{tabular}


Table 4 Total specimens, KUs, and Welcans tabulated for the individual pathologists $(A-G)$

\begin{tabular}{|c|c|c|c|c|c|c|c|c|c|}
\hline & & $A$ & $B$ & $C$ & $D$ & $E$ & $F$ & $G$ & Total \\
\hline \multicolumn{10}{|l|}{ Numbers/weightings } \\
\hline \multirow[t]{3}{*}{ Histology } & KUs & 3252 & 819 & 5553 & 5871 & 7983 & 4771 & 6497 & 28249 \\
\hline & Specimens & 1670 & 690 & 2682 & 2479 & 3530 & 2146 & 3422 & 13197 \\
\hline & Welcans & 95154 & 28520 & 162758 & 189657 & 237825 & 117736 & 217964 & 831650 \\
\hline \multirow{3}{*}{$\begin{array}{l}\text { Non gynaecological } \\
\text { cytology }\end{array}$} & KUs & 3 & 1207 & 32 & 1 & 375 & 123 & 115 & 1741 \\
\hline & Specimens & 3 & 1205 & 33 & 1 & 375 & 123 & 115 & 1740 \\
\hline & Welcans & 189 & 84336 & 2350 & 63 & 27936 & 8432 & 8217 & 123306 \\
\hline \multirow{3}{*}{ Cervical cytology } & KUs & 0 & 3682 & 2276 & 0 & 808 & 896 & 292 & 7662 \\
\hline & Specimens & 0 & 1841 & 1138 & 0 & 404 & 446 & 146 & 3829 \\
\hline & Welcans & 0 & 70590 & 43362 & 0 & 23940 & 17709 & 7275 & 155601 \\
\hline \multirow[t]{3}{*}{ Necropsy } & KUs & 300 & 0 & 720 & 840 & 760 & 610 & 610 & 3230 \\
\hline & Specimens & 30 & 0 & 72 & 84 & 76 & 61 & 61 & 323 \\
\hline & Welcans & 11994 & 0 & 28423 & 19282 & 37625 & 17815 & 26882 & 115139 \\
\hline \multicolumn{10}{|l|}{ Percentage total } \\
\hline \multirow[t]{3}{*}{ Histology } & KUs & 11.5 & 2.9 & 19.7 & 20.8 & 28.3 & 16.9 & 23.0 & 100 \\
\hline & Specimens & 12.7 & 5.2 & 20.3 & 18.8 & 26.7 & 16.3 & 25.9 & 100 \\
\hline & Welcans & 11.4 & 3.4 & 19.6 & 22.8 & 28.6 & 14.2 & 26.2 & 100 \\
\hline \multirow{3}{*}{$\begin{array}{l}\text { Non-gynaecological } \\
\text { cytology }\end{array}$} & KUs & 0.2 & 69.3 & 1.8 & 0.1 & 21.5 & 7.1 & 6.6 & 100 \\
\hline & Specimens & 0.2 & 69.3 & 1.9 & 0.1 & 21.6 & 7.1 & 6.6 & 100 \\
\hline & Welcans & 0.2 & 68.4 & 1.9 & 0.1 & 22.7 & 6.8 & 6.7 & 100 \\
\hline \multirow[t]{3}{*}{ Cervical cytology } & KUs & 0.0 & 48.1 & 29.7 & 0.0 & 10.5 & 11.7 & 3.8 & 100 \\
\hline & Specimens & 0.0 & 48.1 & 29.7 & 0.0 & 10.6 & 11.6 & 3.8 & 100 \\
\hline & Welcans & 0.0 & 45.4 & 27.9 & 0.0 & 15.4 & 11.4 & 4.7 & 100 \\
\hline \multirow[t]{3}{*}{ Necropsy } & KUs & 9.3 & 0.0 & 22.3 & 26.0 & 23.5 & 18.9 & 18.9 & 100 \\
\hline & Specimens & 9.3 & 0.0 & 22.3 & 26.0 & 23.5 & 18.9 & 18.9 & 100 \\
\hline & Welcans & 10.4 & 0.0 & 24.7 & 16.7 & 32.7 & 15.5 & 23.3 & 100 \\
\hline
\end{tabular}

Table 5 Total output of the laboratory tabulated by KUs, specimen numbers, Welcans, and Korner analysis

\begin{tabular}{|c|c|c|c|c|c|c|c|}
\hline & & Histology & $\begin{array}{l}\text { Non-gynaecological } \\
\text { cytology }\end{array}$ & $\begin{array}{l}\text { Cervical } \\
\text { cytology }\end{array}$ & Necropsies & Supervision & Total \\
\hline \multirow[t]{2}{*}{ KUs } & Count & 34746 & 1856 & 7954 & 3840 & 5196 & 53592 \\
\hline & & 64.8 & 3.5 & 14.8 & 7.2 & 9.7 & 100 \\
\hline \multirow[t]{2}{*}{ Specimen number } & Count & 16619 & 1856 & 3977 & 384 & 0 & 22836 \\
\hline & $\%$ & 72.7 & 8.1 & 17.4 & 1.7 & 0.0 & 100 \\
\hline \multirow[t]{2}{*}{ Welcans } & Count & 1049614 & 131523 & 1126882 & 142609 & 0 & 2450628 \\
\hline & $\%$ & 42.8 & 5.4 & 46.0 & 5.8 & 0.0 & 100 \\
\hline \multirow[t]{2}{*}{ Korner } & Count & 83552 & 1692 & 35433 & 7680 & 0 & 128357 \\
\hline & $\%$ & 65.1 & 1.3 & 27.6 & 6.0 & 0.0 & 100 \\
\hline
\end{tabular}

rived from the hospital. Interestingly, the relatively close banding of Welcans, Korner units, and KUs is felt to approximate to the relative workload within the department.

Finally, it is clear that supervision is only assessable in terms of KUs, with Welcans and Korner units not offering any assessment for this area of our activity.

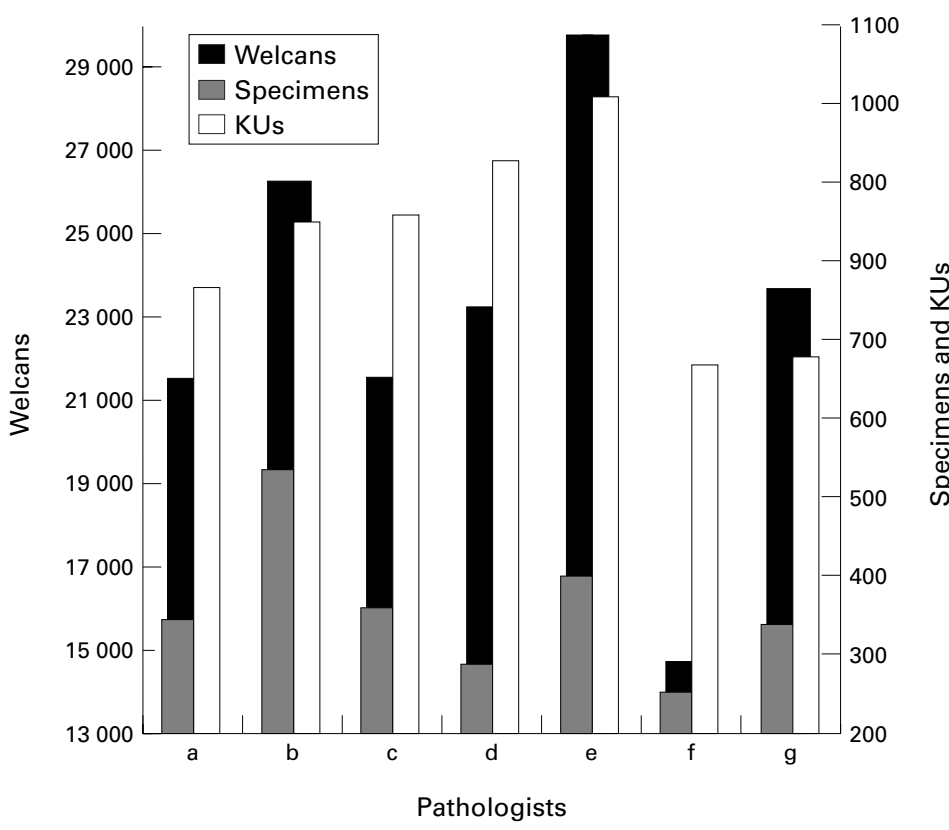

Figure 1 Histogram showing output of seven pathologists in terms of activity per session over a year.
If one chooses to expand the histology, nongynaecological cytology, cervical cytology, and necropsy weightings and to quantitate them in terms of percentage output for the respective pathologists within the department, one can see from table 4 that the numbers produce broadly similar percentages of the total when analysed in the separate areas of work. Thus if one looks at pathologist A one can see that the scores, while differing markedly, have a small range in terms of the total percentages. This is an important point in that it indicates specimens, Welcans, and KUs all appear to be a valid way of calculating activity. However, problems arise when one chooses to combine the totals (specimen numbers, Welcans, KU) for all histopathology/cytopathology activity. The bar histogram (fig 1) shows the output from the seven pathologists in terms of activity per session over the year. The columns for specimen numbers and Welcans show wide fluctuation, with convergence and divergence focally. Given that these results have been produced per session of consultant activity, one would expect them to be comparable, assuming all consultants work equally hard. The KU weighting scheme results in the bar histogram are much more closely distributed. Furthermore, since the department had a retirement and a gap before the next post holder arrived, it is not surprising that some values over this year appear less than others. Thus it is refreshing to see that the department's perception of its workload is reflected so precisely in the graph. 
Dividing the total KU activity by the number of sessions employed within the department in a previous year allowed us to derive an average KU consultant weighting per session of 820 $\mathrm{KU}$. This figure is one of the cornerstones of calculation within our department. Specifically, were an increase number of biopsies to arrive, it will follow that an increased KU score will be produced. Over time, the increased workload experienced by the department has been translated into a successful and specific request for increased funding in terms of WTE (whole time equivalent) consultants.

\section{Discussion}

Previous attempts to calculate the workloads of histopathologists or histopathology departments have used many strategies. The simplest involves counting the number of specimens or cases per year and dividing this number by the pathologists within the department. While crude, if the pathologists allocate the work equally and if the specimen type remains broadly constant, this calculation can serve to provide retrospective information.

Attempts to standardise laboratory activity initially used Korner statistics, ${ }^{1}$ although more recently Welcans ${ }^{2}$ have been favoured. The Korner weightings appear to offer little in terms of histopathology laboratory workload calculations. When they were introduced, Welcans were at first thought to produce a more reliable analysis of department activity. However, the calculation was derived from technical activity and there was a presumption that cases with a high technical input also took up an equally high proportion of pathologist's time. An example of the imbalance would be an $H \& E$ stained section required for an appendix (1 Welcan) compared with a PAS stain for fungi (17 Welcans). The results of the departmental activity expressed in Korner and Welcan weightings (tables 4 and 5) clearly indicate the wide ranges possible within a single department, and the analysis per pathologist sessional activity indicates that both systems are flawed.

In an attempt to address individual pathologists' activity, recently the Royal College of Pathologists has suggested counting the number of slides or sections studied per unit time as a refinement upon case numbers. ${ }^{3}$ However, this enhancement still fails to address the pathologists' input in specific specimen groups. In an ideal world, a departmental computer could allocate a difficulty rating according to the type of case, building in separate variables reflecting the number of sections, the number of immunohistochemical stains and other tests, plus or minus the ultimate SNOMED diagnostic code as separate factors. This degree of precision, while laudable, may represent overkill though it could serve as the goal for the future.

We have found the KU weighting analysis simple to apply and particularly useful for demonstrating the work expended by the department on a yearly basis. That the results can be examined in terms of individual pathologists and groups of specimens is an obvious advantage, allowing trends to be discerned promptly and thus affording the department the opportunity to plan for the future. We have on occasion considered individual clinical management teams/ directorates with the individual consultant teams being displayed against the number of specimens across the year for even finer examination of departmental trends. Overall, the $\mathrm{KU}$ analysis has proved reliable, robust, and versatile within our department.

Perhaps the most valuable figure derived from this analysis was the average KU output per session of consultant time- $820 \mathrm{KU}$. This result, derived from an earlier year's work, has provided a benchmark against which to measure individual pathologists' activities, clinical directorate output, and planned service developments.

The protocol illustrated by this department can easily be extrapolated into practice within any district general hospital, although the supervision row of junior pathologists' cases would not be applicable. For university departments, one could assign KU activity for whole sessions of research, teaching, or management purposes, although the figure of $820 \mathrm{KU}$ per session can be used as a ready reckoner on a direct activity per time basis.

It must be stressed that the evaluation provided is based on our unit's interpretation of pathology workload, and that while focusing on consultant activity it also roughly reflects the laboratory work since the KU score reflects the number of sections, stains, and blocks taken. We believe the assignment of KU weights to be fair, although we recognise that other centres may disagree with some of the values that have been derived. Rather than suggest that each centre could define its own workload weight, we would be keen to hear the results of this scheme's application to other centres, thus perhaps allowing pathologists to titrate some of the weightings on a national basis if this methodology was accepted.

However, we stress that the key impact of this analysis resides in its ability to predict service developments within an individual hospital. For example, introduction of an extra gastroenterology endoscopy list, where an average list may generate three gastric biopsies, two duodenal biopsies, and one oesophageal biopsy ( $9 \mathrm{KU})$, will produce a totalled yearly increase in activity of $468 \mathrm{KU}$. This equates to 0.57 extra consultant WTE sessions, thereby providing an accurate way of predicting and costing this service development. Alternatively, a new appointment within an established field, such as surgery, allows one to extract the previous years' activity within the field in question to calculate the impact of a new surgeon with the same interest within the hospital. We have projected that a new consultant surgeon at our centre would generate a total of 1272 KUs extra activity per year, requiring a further 1.55 sessions of histopathologist WTE activity.

We have chosen to publicise our department's methodology in order, first, to ask other departments to apply it and to advise us as to the benefits or otherwise that they find with this scoring scheme. In the second place, we 
consider that Welcans and other methods of calculating workload are now entering the arena for review and we hope that our analysis is a step forward in titrating work against specimen type on an individual and departmental basis. We look forward to constructive criticism and comment on this scheme.

We are grateful to our colleagues, Drs M Balsitis, J L Channer, A Kennedy, J R Shortland, and J H F Smith for their support.
We are also grateful to Mrs J Galloway, Ms K Snee, Mrs A Carver, and Mrs S Woodward for support and understanding during the introduction of this programme and the preparation of the manuscript.

1 DHSS Health Service Indicators Group. A report on Korner indicators. London: HMSO, 1988.

2 Seaton B, Croft E. Pathology workload measurement and interpretation. Principles of workload measurement. $I M L S$ Gazette, April 1990:187-8.

3 Consultant staffing and workload of histopathology departments. Bull R Coll Pathol 1997;98:1-3.

\section{Royal College of Pathologists Forthcoming symposia}

Joint Royal College of Pathologists and Armed forces Institute of Pathology meeting:

Diagnostic Surgical Pathology

St John's College, Cambridge, 30 July to 2 August 1998

Diabetes Mellitus

Royal College of Pathologists, London, 30 September 1998

The British Association of Urological Surgeons and the Royal College of Pathologists:

Uropathology-Debate and Controversy

Edinburgh International Conference Centre, 6-7 October 1998

Fetal, Neonatal and Childhood Haematology-Paradoxes, Problems and Progress Royal College of Pathologists, London, 28 October 1998

Further details from:

Scientific Meetings Officer, Royal College of Pathologists,

2 Carlton House Terrace, London SW1Y 5AF;

tel +44 (0)171 9305862 , extension $24 / 25$;

fax +44(0)171321 0523 . 